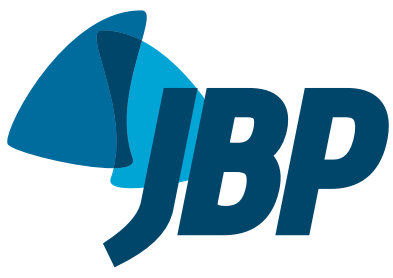

\title{
Should we use prognostic scores for acute pulmonary thromboembolism in clinical practice?
}

\author{
Marcelo Basso Gazzana ${ }^{1,2,3, a}$, Igor Gorski Benedetto ${ }^{1,2,3, b}$
}

Acute pulmonary thromboembolism (PTE) is a lifethreatening disease, the incidence of which has increased in recent years; however, the mortality of acute PTE has decreased, possibly due to improved diagnostic and treatment strategies. $^{(1)}$ In this context, establishing a prognosis is essential for patient management.

Risk stratification has long been used in the management of acute conditions and acute exacerbations of chronic diseases, including myocardial infarction, stroke, and asthma exacerbation. The objectives of stratifying patients into risk groups are as follows: to inform patients about the course of their disease; to identify lower-risk patients who can be discharged early or even receive home treatment (given that novel oral anticoagulants such as apixaban and rivaroxaban do not require a heparin bridge); to identify higher-risk patients requiring treatments that are more aggressive, such as exogenous thrombolysis and embolectomy; to select patients for clinical drug trials; and to compare hospitals by means of severity-adjusted quality health care outcomes. ${ }^{(2)}$

Hemodynamic instability is the strongest predictor of the outcome of acute PTE. However, most patients are normotensive and constitute a heterogeneous group, other variables being required for risk stratification. Although echocardiographic variables (showing right ventricular dysfunction) and levels of biomarkers (such as troponin and natriuretic peptides, showing myocardial injury or stress) are primarily used in daily practice, the major guidelines on acute PTE recommend that prognostic scores be used after hemodynamic assessment. ${ }^{(3,4)}$ Any new score requires the following: 1) derivation; 2) validation in a different population; and 3) study of its clinical impact. ${ }^{(2)}$

The Pulmonary Embolism Severity Index (PESI), comprising 11 variables and 5 risk classes (ranging from I to V), and the simplified PESI, comprising 6 variables and 2 risk classes (i.e., low risk and high risk), are among the most widely studied and validated prognostic scores for acute PTE. A PESI risk class of I or II indicates a lowrisk population (as does a simplified PESI of zero), the 30-day mortality rate being less than 3\%. . $^{(5,6)}$ The PESI and the simplified PESI can identify a low-risk population in $45 \%$ of patients with acute PTE and reduce the length of hospital stay without the need for additional tests and without increasing the risk of death, recurrent PTE, or severe bleeding. Evaluation is complemented by The Hestia Study criteria for outpatient treatment. ${ }^{(7)}$ In a meta-analysis of 71 studies (a total of 44,298 patients), prognostic scores were shown to be valid and useful for identifying low-risk patients. ${ }^{(8)}$ Therefore, patients can be treated at home safely and efficiently. $(3,9)$

It should be noted that the PESI has a high negative predictive value but a low positive predictive value. ${ }^{(10)}$ This means that the PESI does not adequately identify high-risk patients among normotensive patients requiring intensive monitoring and, in some cases, treatments that are more aggressive. Other scores are more appropriate for this purpose, including the Bova score (a systemic blood pressure of $90-100 \mathrm{mmHg}$, elevated troponin levels, right ventricular dysfunction as assessed by echocardiography or $\mathrm{CT}$, and a heart rate $\geq 110 \mathrm{bpm}$ ); Prognostic Factors for Pulmonary Embolism, including altered mental status, cardiogenic shock on admission, cancer, serum brain natriuretic peptide (BNP) levels, and right ventricular/left ventricular ratio as assessed by echocardiography; and the Heart-type Fatty Acid-Binding Protein, Syncope, and Tachycardia score. In addition, a clinical prognostic rule should be used in order to predict the risk of intracranial bleeding in patients undergoing thrombolytic therapy, a borderline risk-benefit ratio being taken into account in normotensive patients with right ventricular dysfunction alone. ${ }^{(1)}$ Figure 1 shows a management algorithm based on a risk stratification algorithm presented in a nonsystematic review published recently. ${ }^{(2)}$ It should be noted that this is a practical approach based on independent clinical studies; it has yet to be validated as a viable strategy.

In the current issue of the JBP, Soriano et al. ${ }^{(12)}$ published a single-center study aimed at validating the original and simplified versions of the PESI in a historical cohort of patients in Brazil in order to predict 30-day mortality following acute PTE. The authors retrospectively evaluated 123 patients admitted to the emergency department of a public, tertiary referral hospital that exclusively serves patients requiring acute care. They concluded that the PESI can predict 30-day mortality, the original version being more accurate than the simplified version.

Certain points should be noted. Patients with acute PTE were identified on the basis of International Classification of Diseases, 10th revision (ICD-10) codes on discharge records. However, ICD-10 codes have low sensitivity for identifying patients with acute PTE. ${ }^{(13)}$ Therefore, it is possible that some patients (particularly low-risk patients) were missed and not included in the study, a possibility that might explain the high proportion of patients with cardiogenic shock. It is also of note that the

1. Serviço de Pneumologia e Cirurgia Torácica, Hospital Moinhos de Vento, Porto Alegre (RS) Brasil.

2. Serviço de Pneumologia, Hospital de Clínicas de Porto Alegre, Porto Alegre (RS) Brasil.

3. Programa de Pós-Graduação em Ciências Pneumológicas, Universidade Federal do Rio Grande do Sul, Porto Alegre (RS) Brasil.

a. (iD) http://orcid.org/0000-0003-0086-1890; b. (iD) http://orcid.org/0000-0001-6527-9805 


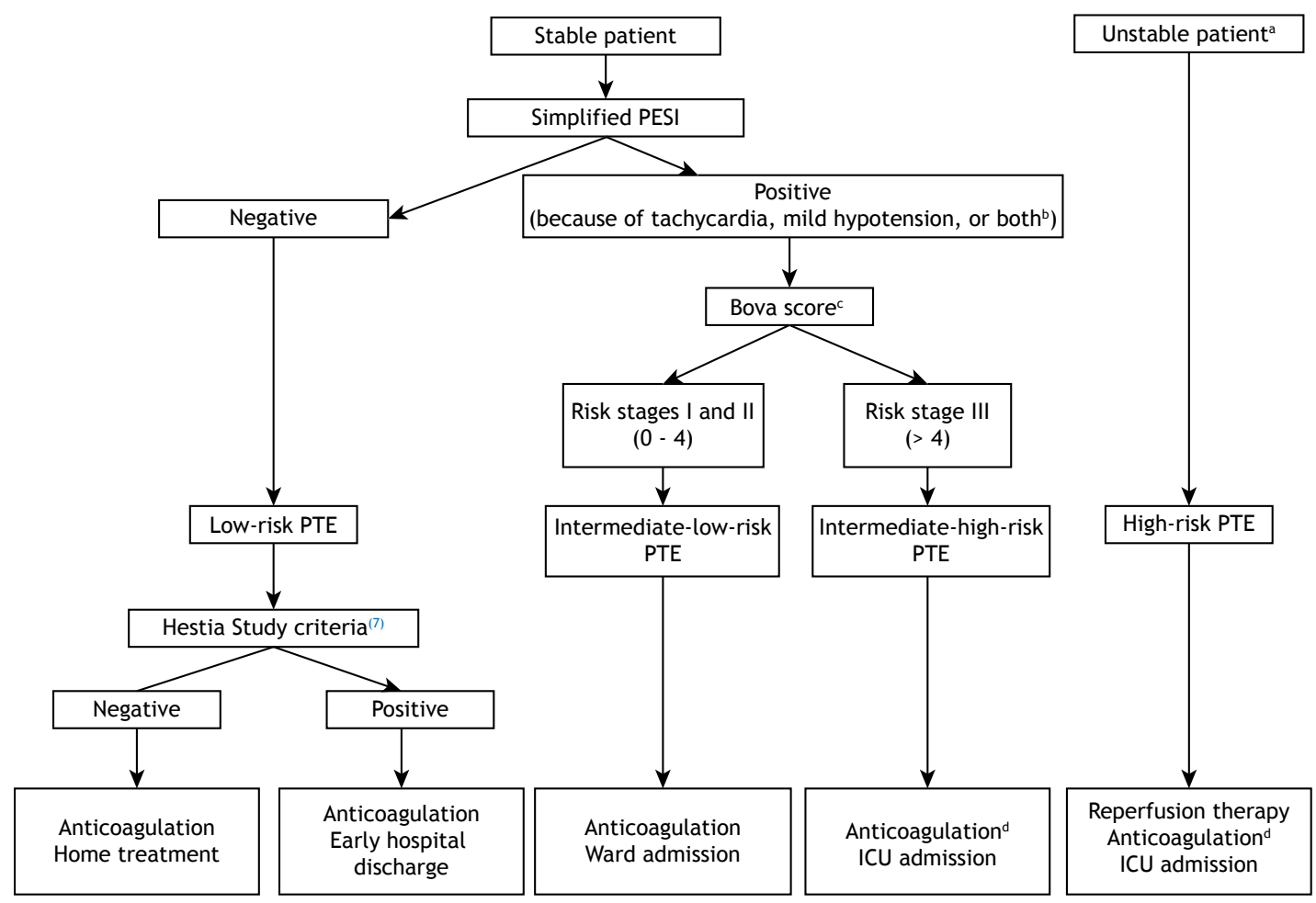

Figure 1. Treatment algorithm based on risk stratification. PESI: Pulmonary Embolism Severity Index; and PTE: pulmonary thromboembolism. aUnstable patient: a systemic systolic blood pressure of $<90 \mathrm{mmHg}$ or a $40-\mathrm{mmHg}$ drop lasting longer than 15 min and not caused by new arrhythmias, hypovolemia, or sepsis; or cardiogenic shock (reduced cardiac output associated with signs of tissue hypoperfusion, including oliguria, decreased level of consciousness, decreased skin perfusion, and lactic acidosis). bMild hypotension: a systemic systolic blood pressure of 90-100 mmHg. ${ }^{c} \mathrm{The}$ Bova score includes the following variables: biomarker (troponin) levels and right ventricular dysfunction as assessed by echocardiography or chest CT angiography. dUnfractionated heparin should be the preferred anticoagulation strategy in such cases. Adapted from Morillo et al.(2)

study evaluated patients who had been hospitalized for acute PTE. It did not include patients who had been hospitalized for other reasons and had acute PTE during their hospital stay (a condition known as secondary pulmonary embolism or hospital-acquired pulmonary embolism). This reduces the external validity of the results.

Despite the limitations inherent to studies of historical data conducted at highly specialized centers, the study by Soriano et al. ${ }^{(12)}$ is relevant and provides national data that can bridge the gap between clinical studies and the daily practice of pulmonology in Brazil. In patients with an established diagnosis of acute PTE, it is important to stratify the risk of poor outcome, clinical prognostic scores being useful for this purpose. They are also useful in selecting the most appropriate therapy for a given patient. However, prospective randomized studies focusing on patient management (risk stratification-guided therapy) are needed for external validation of this concept and, consequently, a higher level of evidence to strengthen recommendations for the management of patients with acute PTE in Brazil.

\section{REFERENCES}

1. Jiménez D, de Miguel-Díez J, Guijarro R, Trujillo-Santos J, Otero $R$, Barba $R$, et al. Trends in the Management and Outcomes of Acute Pulmonary Embolism: Analysis From the RIETE Registry. J Am Coll Cardiol. 2016;67(2):162-170. https://doi.org/10.1016/j. jacc.2015.10.060

2. Morillo R, Moores L, Jiménez F. Prognostic Scores for Acute Pulmonary Embolism. Semin Thromb Hemost. 2017;43(5):486-492. https://doi.org/10.1055/s-0036-1597287

3. Howard LS, Barden S, Condliffe R, Connolly V, Davies C, Donaldson $J$, et al. British Thoracic Society Guideline for the initial outpatient management of pulmonary embolism. BMJ Open Respir Res. 2018;5(1):e000281. https://doi.org/10.1136/bmjresp-2018-000281

4. Konstantinides SV, Torbicki A, Agnelli G, Danchin N, Fitzmaurice D Galiè N, et al. 2014 ESC guidelines on the diagnosis and management of acute pulmonary embolism. Eur Heart J. 2014 35(43): 3033-69, 3069a-3069k.

5. Aujesky D, Obrosky DS, Stone RA, Auble TE, Perrier A, Cornuz J, et al. Derivation and validation of a prognostic model for pulmonary embolism. Am J Respir Crit Care Med. 2005;172(8):1041-6. https:// doi.org/10.1164/rccm.200506-8620C

6. Jiménez D, Aujesky D, Moores L, Gómez V, Lobo JL, Uresandi F, et al. Simplification of the pulmonary embolism severity index for prognostication in patients with acute symptomatic pulmonary embolism. Arch Intern Med. 2010;170(15):1383-9. https://doi. org/10.1001/archinternmed.2010.199

7. Zondag W, Mos IC, Creemers-Schild D, Hoogerbrugge AD, Dekkers OM, Dolsma J, et al. Outpatient treatment in patients with acute pulmonary embolism: the Hestia Study. J Thromb Haemost. 2011;9(8):1500-7. https://doi.org/10.1111/j.1538-7836.2011.04388.x

8. Elias A, Mallett S, Daoud-Elias M, Poggi J, Clarke M. Prognostic models in acute pulmonary embolism: a systematic review and metaanalysis. BMJ Open 2016;6(4):e010324. https://doi.org/10.1136/ 
bmjopen-2015-010324

9. Aujesky D, Roy PM, Verschuren F, Righini M, Osterwalder J, Egloff $M$, et al. Outpatient versus inpatient treatment for patients with acute pulmonary embolism: an international, open-label, randomised, noninferiority trial. Lancet. 2011;378(9785):41-8. https://doi.org/10.1016/ S0140-6736(11)60824-6

10. Meyer G, Planquette B, Sanchez O. Risk stratification of pulmonary embolism: clinical evaluation, biomarkers or both? Eur Respir $\mathrm{J}$. 2015;46(6):1551-3. https://doi.org/10.1183/13993003.01562-2015

11. Barrios D, Morillo R, Yusen RD, Jiménez D. Pulmonary embolism severity assessment and prognostication. Thromb Res 2018;163:246-251. https://doi.org/10.1016/j.thromres.2017.09.007

12. Soriano LA, Castro TT, Vilalva K, Borges MC, Pazin-Filho A, Miranda $\mathrm{CH}$. Validation of the Pulmonary Embolism Severity Index for risk stratification after acute pulmonary embolism in a cohort of patients in Brazil. J Bras Pneumol. 2019;45(1):e20170251

13. Zhan C, Battles J, Chiang YP, Hunt D. The validity of ICD-9-CM codes in identifying postoperative deep vein thrombosis and pulmonary embolism. Jt Comm J Qual Patient Saf. 2007;33(6):326-31. https:// doi.org/10.1016/S1553-7250(07)33037-7 